\title{
Measurements of the very-forward energy in pp collisions at the LHC and constraints for cosmic ray air showers
}

\author{
Sebastian Baur* \\ IIHE, Université Libre de Bruxelles \\ E-mail: sebastian.baureulb.ac.be \\ Ralf Ulrich \\ IKP, Karlsruhe Institute of Technology \\ E-mail: ralf.ulrich@kit.edu

\section{for the CMS collaboration}

\begin{abstract}
The very-forward energy production in hadron collisions is of great importance for the understanding of ultra-high energy cosmic ray air showers. The CASTOR calorimeter of CMS is located at $-6.6<\eta<-5.2$ in a unique phase-space for this purpose; the characteristics of secondaries in this phase-space of hadron collisions have a determining impact on the formation of air shower cascades. We present the latest results of CASTOR including the first direct measurement of the ratio of electromagnetic to hadronic energy in the forward phase-space at the LHC. Possible implications of these results for cosmic ray physics are outlined.
\end{abstract}

36th International Cosmic Ray Conference -ICRC2019-

July 24th - August 1st, 2019

Madison, WI, U.S.A.

${ }^{*}$ Speaker. 


\section{Introduction}

Particle production at forward rapidities in high energy hadron collisions has crucial impact on the development of extensive air showers in the atmosphere [1]. The underlying production mechanisms, however, can - to a large extent - not be accurately described by calculations from first principles. The most relevant processes, especially multiparton interactions (MPI) and beam remnant fragmentations, are therefore modelled phenomenologically in Monte Carlo event generators. Free parameters in these models are tuned to experimental data [2, 3].

A large variety of measurements at hadron colliders is therefore necessary to ensure a best possible understanding of the particle production in air showers. Data from the CERN LHC has proven essential in reducing model-related uncertainties in the prediction of air showers at ultrahigh energies $[4,5]$.

The CASTOR calorimeter of the CMS detector is ideally suited to contribute relevant constraints for very-forward particle production and subsequently for the understanding of air shower developments. In this contribution, we review measurements performed with proton-proton collisions at a center-of-mass energy of $13 \mathrm{TeV}$ at the CERN LHC and outline possible implications for hadronic event generators used for air shower simulations.

\section{The CASTOR calorimeter of CMS}

The CASTOR calorimeter of the CMS experiment is a sampling calorimeter composed of layers of fused silica quartz plates and tungsten absorbers. It is located on the negative side of CMS and covers the pseudorapidity region $-6.6<\eta<-5.2$. CASTOR is composed of two subsections: the two channels closest to the interaction region have a combined depth of 20 radiation lengths and form the electromagnetic section. The remaining 12 channels constitute the hadronic section. The full depth of the calorimeter amounts to 10 hadronic interaction lengths. The energy deposited in the electromagnetic section is dominated by the energy loss of electrons and photons. Since CASTOR is located about $14 \mathrm{~m}$ away from the interaction region, neutral pions decay before they reach the detector and contribute to the energy measured in the electromagnetic section. Due to its design, CASTOR can not detect single particles, but measures the combined energy of all particles arriving at the detector. It is, however, possible to separate the energy in the two subsections and therefore study the energy of electrons and photons independently from the one of hadrons. A more detailed description of the CMS detector and the CASTOR calorimeter is given in Refs. $[6,7,8]$.

\section{Energy measurements with CASTOR}

During the startup period of the LHC Run 2 in 2015, CASTOR took data of proton-proton collisions at the current record center-of-mass energy of $13 \mathrm{TeV}$. In this period, LHC was operating with very small beam intensities and therefore provided very pure conditions to measure veryforward particles without interference from additional pileup collisions. The solenoid magnet of CMS was turned off during this period, which reduced the impact of stray fields on the calorimeter, increasing the experimental resolution compared to previous data taking periods.

The data are compared to predictions of the relevant interaction models used to describe high energy hadronic collisions like Pythia8 [9, 10, 11] and HeRwig7 [12, 13], as well as those 

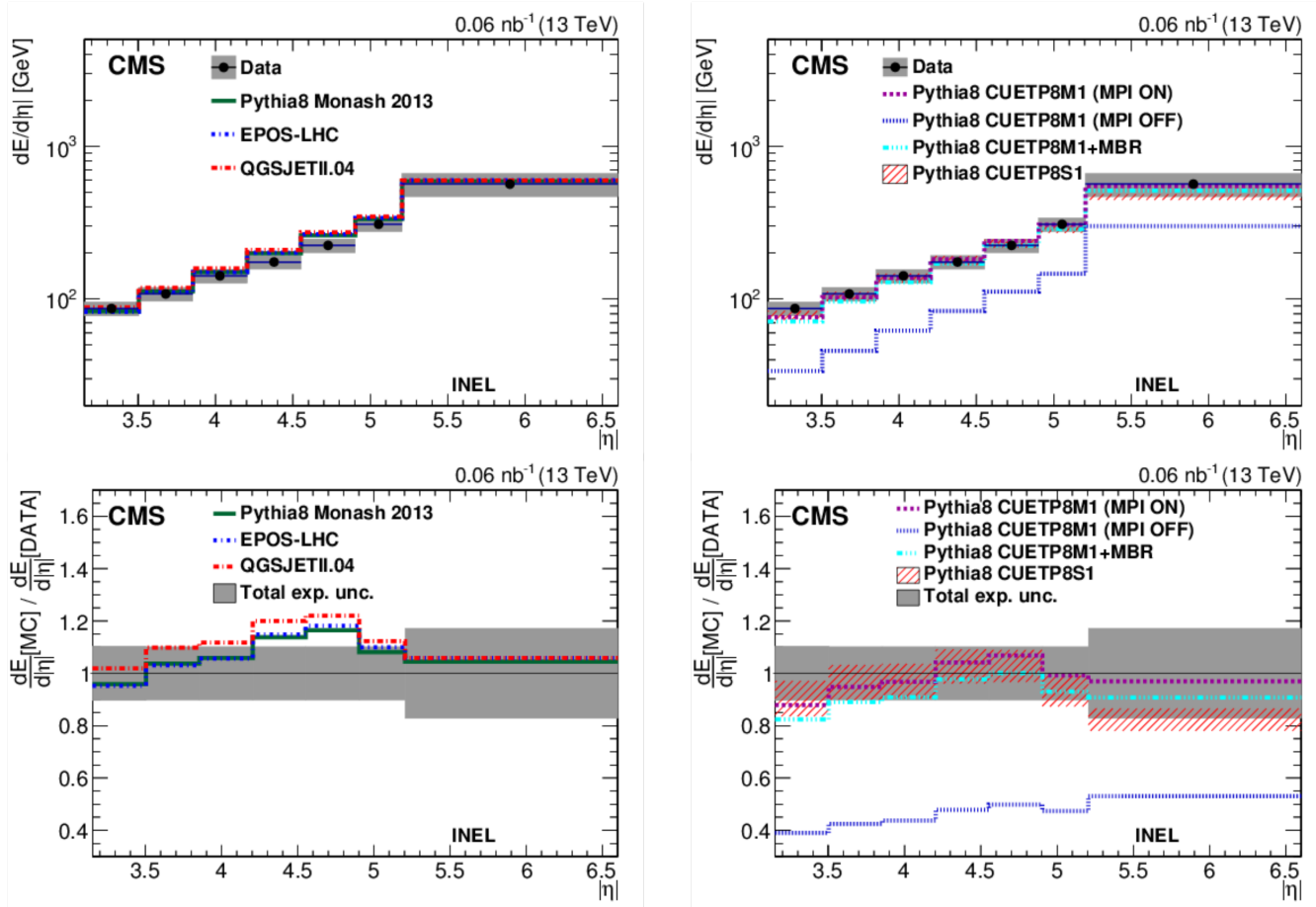

Figure 1: Energy density at particle level as a function of pseudorapidity at $13 \mathrm{TeV}$ for compared to predictions of various event generators. The grey band shows the total systematic uncertainty correlated across pseudorapidity bins. The bottom panel shows the models with respect to the data [19].

commonly used for the simulation of cosmic ray air showers, such as EPOS [14, 5], QGSJETII [15], and SIBYLL $[16,17,18]$.

All measurements discussed in this contribution share a common event selection, which is tuned to be as inclusive as possible and - at the same time - to reproduce the detector coverage. On detector level, a minimal energy deposition of $5 \mathrm{GeV}$ is required in the Hadron Forward (HF) calorimeters of CMS, which cover the range $3.15<|\eta|<5$.2. On the particle level, this selection is reproduced by separating the final state particles into two subsystems with respect to the largest rapidity gap. The ratio of the larger mass divided by the squared center-of-mass energy is the proton momentum loss $\xi$, which is required to be $\xi>10^{-6}$, see Ref.[19] for details. Diffractive events with very low masses in the diffractive system(s) are therefore excluded in these analyses.

\subsection{Pseudorapidity-dependent forward energy density}

The average energy density per collision and unit in pseurorapidity is measured as a function of the pseudorapidity using the CASTOR and the Hadron Forward (HF) calorimeter of CMS. The covered range extends from $3.15<|\eta|<6.6$ [19]. Fig. 1 shows the results as published in Ref. [19].

The most accurate predictions are provided by PYTHIA8 with tune CUETP8M1. The other interaction models show overall good agreement with the data, slightly overestimating the data in parts of the studied pseudorapidity region. The relative change of the energy with $\eta$, however, is not well described by all models, which exhibit a too strong increase in the region covered by the HF calorimeter within $3.15<|\eta|<5.20$. For Pythia8 CUETP8S1, the tune uncertainties are 

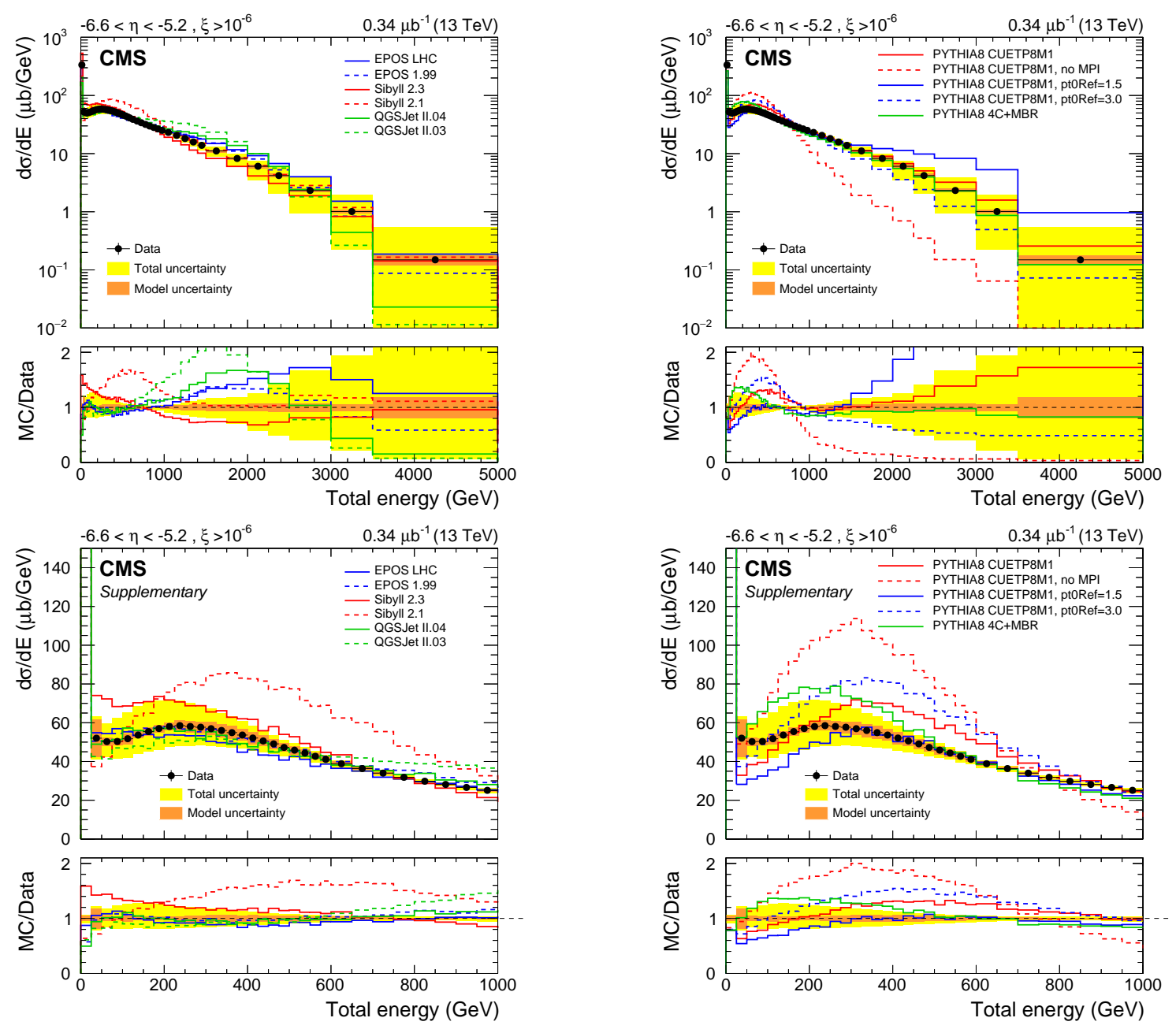

Figure 2: Differential cross section as a function of the total energy in $-6.6<\eta<-5.2$. The left panel shows the data compared to MC event generators mostly developed for cosmic ray induced air showers, and the right panel to different PYTHIA8 tunes. The bottom panel shows the same data with linear scale at low energies. The yellow band indicates the total uncertainty of the measurement, the orange band the model-uncertainty due to the unfolding [20].

illustrated by a red band in the right panels of Fig. 1. These uncertainties are smaller than the spread of the different models. Therefore, inherent differences of these models are resolved. Furthermore, it is observed, that the modelling of MPI is essential to describe the data.

\subsection{Forward energy spectra}

The detailed energy distribution within the CASTOR acceptance is studied in more detail in Ref. [20]. The results in terms of the differential cross sections as a function of energy are shown in Figs. 2 and 3. This measurement is performed as a function of the total energy (Fig. 2), as well as of the electromagnetic and hadronic components (Fig.3) individually. This represents the first measurement in which the electromagnetic and hadronic energies are investigated separately with the CASTOR calorimeter. 

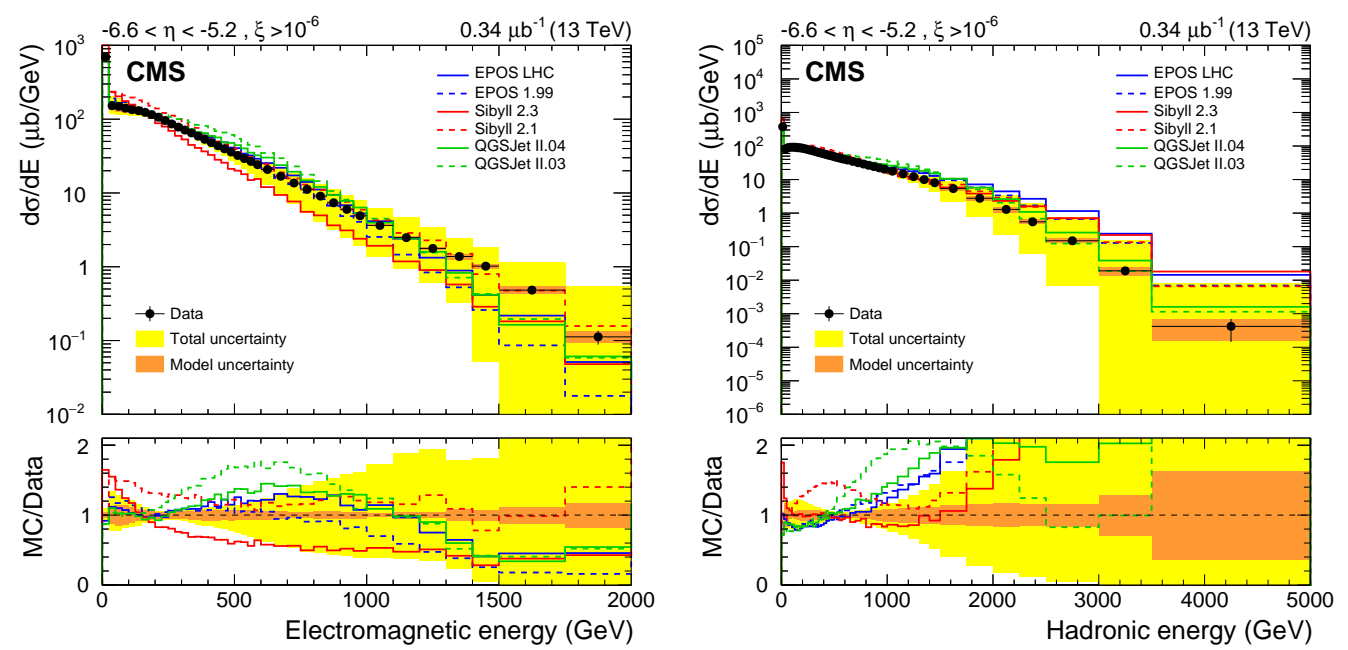

Figure 3: Differential cross section as a function of the electromagnetic energy (left) and the hadronic energy (right). The yellow band indicates the total uncertainty of the measurement, the orange band the model-uncertainty due to the unfolding [20].

The importance of MPI modelling is demonstrated by changing of the parameter $p_{\mathrm{T}, 0}^{\mathrm{ref}}$ of PYTHIA8. The spectral shape changes significantly with changed parameters. This emphasizes the fact that the provided data can be of great value for future parameter tunes. Furthermore, the overall collision elasticity and the amount of diffraction influences the spectral shape at low energies. It can, for example, be observed that the fraction of events with little energy is significantly overestimated by SIBYLL 2.3 compared to the data (see bottom panel of Fig. 2). This can be a hint of a too large elasticity in the model. The separation into the electromagnetic and hadronic contribution provides additional constraints for the models. While the electromagnetic energy spectrum is well described by the latest generation of interaction models, the hadronic energy is slightly overestimated at the edge of the uncertainties.

\subsection{Forward energy in correlation to the central particle multiplicity}

The most recent measurement studies the average energy deposited in CASTOR in correlation to the charged particle multiplicity in the central acceptance of CMS [21]. For this purpose, a modified tracking algorithm is used in order to compensate for the missing magnetic field. Straight lines are reconstructed with the CMS pixel tracker. For this analysis, the detector-level observables are not corrected to the particle level. A forward-folding technique is used instead in order to compare the data to various model predictions. More details on this method are described in Ref. [21]. The results are shown in Figs. 4 and 5. A clear correlation of central particle multiplicity and very-forward energy is found, following the typical shape of the underlying event: the forward energy increases with increasing particle multiplicity. Most of the interaction models predict the average energies accurately within uncertainties. The dominant systematic uncertainty is the CASTOR energy scale which can be eliminated when only the relative increase with multiplicity is studied (see bottom panel of Fig. 4). The data indicate a softer rise than predicted by most models. Both versions of SIBYLL, however, describe the shape very accurately. 

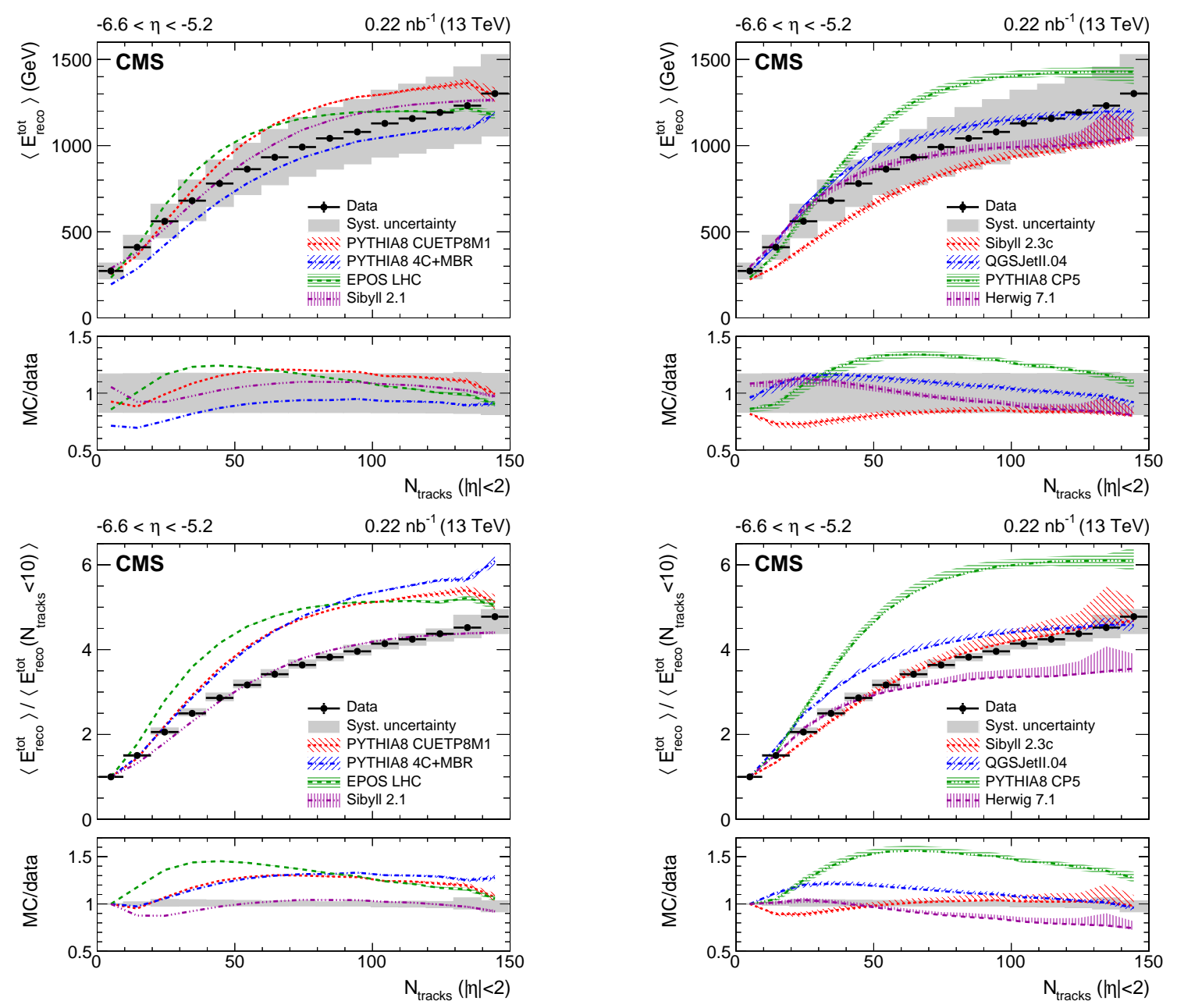

Figure 4: Average energy reconstructed in the CASTOR calorimeter as a function of the number of reconstructed tracks within $|\eta|<2$ (top panel) and normalised to that in the first bin $\left(N_{\mathrm{ch}}<10\right)$ (bottom panel). The data are shown as black markers and the corresponding systematic uncertainties with a gray band, the bands associated with the model predictions illustrate the model uncertainty. [21]

Also here, by using the segmentation of CASTOR, the electromagnetic and hadronic energies are studied independently as well. The ratio of the average electromagnetic and hadronic energy is shown in Fig. 5 as a representative example. In accordance to what is observed in the energy spectra discussed above, the models tend to overestimate the hadronic energy with respect to the electromagnetic and therefore exhibit a lower ratio compared to the data. Most of the models still agree with the measurement within uncertainties. The largest disagreement, not covered by the uncertainties, in found for PyTHIA8 tune CP5 and SibYLL2.3C.

Regarding the still unsolved deficit of muons in air shower simulations [22, 23], the data provide important constraints. Increasing the production of forward hadrons and the energy they carry, could be an effective way to increase the amount of energy available for muon production. The data, however, indicate that there is no room to increase the hadronic energy in forward direction. 

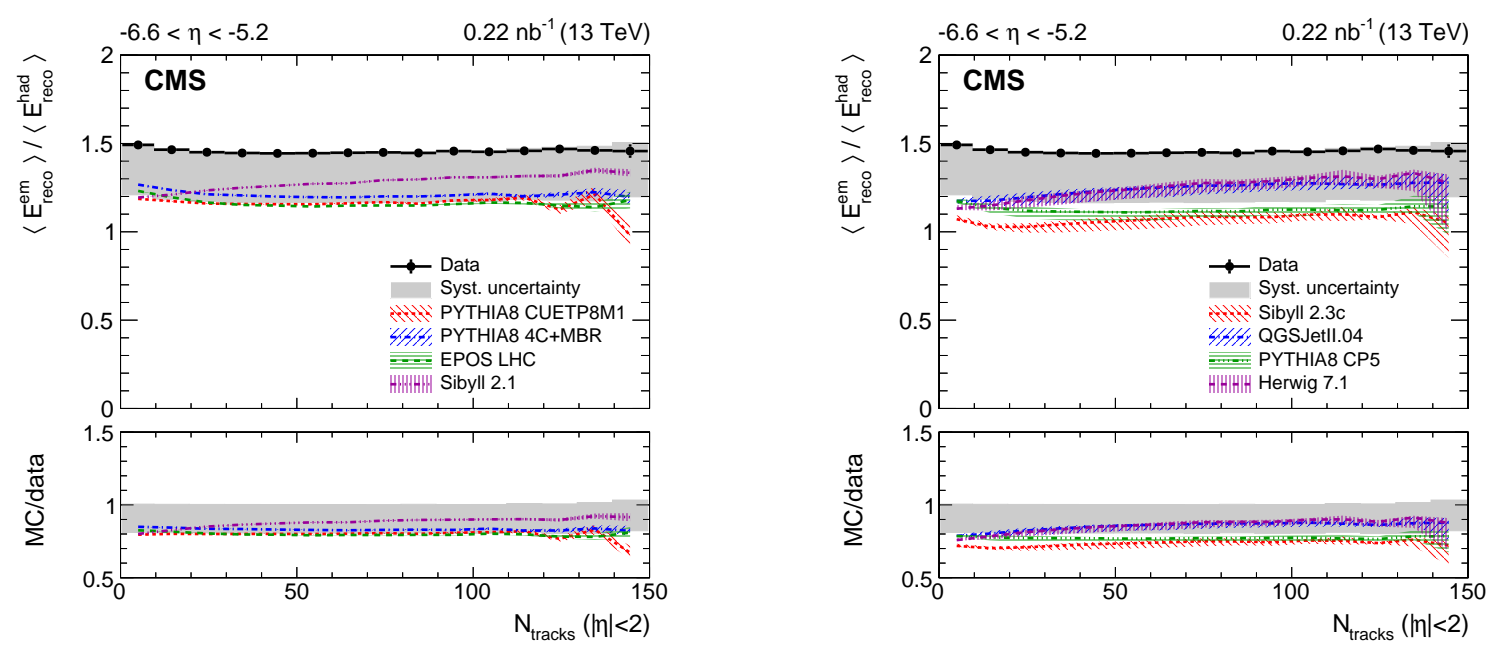

Figure 5: Ratio of the average electromagnetic and hadronic energies reconstructed in the CASTOR calorimeter as a function of the number of reconstructed tracks within $|\eta|<2$. The data are shown as black markers and the corresponding systematic uncertainties with a gray band, the bands associated with the model predictions illustrate the model uncertainty. [21]

\section{Summary}

The CASTOR calorimeter of CMS is a unique detector in the forward phase space. A series of measurements was performed with proton-proton collisions of $\sqrt{s}=13 \mathrm{TeV}$, resulting in detailed descriptions of the energy density and distribution in the forward phase space. The energy is measured inclusively as well as in correlation to the particle multiplicity at central rapidities. Furthermore, the first measurements separating the contribution of electromagnetic and hadronic energy in this phase space were performed. The presented results, therefore, represent benchmark tests for hadronic event generators - in particular those used to describe extensive air showers. Most importantly, the presented data show good sensitivity to the modelling of multiparton interactions in hadronic event generators. The dedicated measurement of the electromagnetic and hadronic energy allows, in addition, to constrain the amount of energy carried by hadrons. This results in strong implications for the modelling of muon production in extensive air showers. Overall, the presented data provide crucial and unique input for future model improvements and an improved understanding of air shower characteristics.

\section{References}

[1] R. Ulrich, R. Engel, and M. Unger, Phys. Rev. D 83 (2011) 054026.

[2] R. Corke and T. Sjostrand, JHEP 03 (2011) 032.

[3] P. Skands, S. Carrazza, and J. Rojo, Eur. Phys. J. C 74 (2014) 3024.

[4] D. d'Enterria, R. Engel, T. Pierog, S. Ostapchenko, and K. Werner, Astropart. Phys. 35 (2011) 98.

[5] T. Pierog, I. Karpenko, J. M. Katzy, E. Yatsenko, and K. Werner, Phys. Rev. C 92 (2015) 034906.

[6] CMS Collaboration, JINST 3 (2008) S08004. 
[7] V. Andreev et al., Eur. Phys. J. C 67 (2010) 601.

[8] CMS-CASTOR Collaboration, P. Göttlicher, Nucl. Instrum. Meth. A 623 (2010) 225.

[9] T. Sjöstrand et al., Comput. Phys. Commun. 191 (2015) 159.

[10] CMS Collaboration, Eur. Phys. J. C 76 (2016) 155.

[11] CMS Collaboration, arXiv:1903.12179.

[12] J. Bellm et al., Eur. Phys. J. C 76 (2016) 196.

[13] S. Gieseke, F. Loshaj, and P. Kirchgaeßer, Eur. Phys. J. C 77 (2017) 156.

[14] K. Werner, B. Guiot, I. Karpenko, and T. Pierog, Phys. Rev. C 89 (2014) 064903.

[15] S. Ostapchenko, Phys. Rev. D 83 (2011) 014018.

[16] E.-J. Ahn, R. Engel, T. K. Gaisser, P. Lipari, and T. Stanev, Phys. Rev. D80 (2009) 094003.

[17] F. Riehn, R. Engel, A. Fedynitch, T. K. Gaisser, and T. Stanev, P o S ( ICRC2015) 558 (2016).

[18] F. Riehn, H. P. Dembinski, R. Engel, A. Fedynitch, T. K. Gaisser, and T. Stanev, POS (ICRC2017) 301 (2018).

[19] CMS Collaboration, Eur. Phys. J. C 79 (2019) 391.

[20] CMS Collaboration, JHEP 08 (2017) 046.

[21] CMS Collaboration, arXiv:1908.01750.

[22] Pierre Auger Collaboration, Phys. Rev. D 91 (2015) 032003. Erratum: Phys. Rev. D 91 (2015) 059901.

[23] Pierre Auger Collaboration, Phys. Rev. Lett. 117 (2016) 192001. 